\title{
Nahrungsmittelallergie bei Kindern: Ferienlager trainiert Selbstsicherheit
}

\begin{abstract}
Nahrungsmittelallergien im Kindesalter haben einen maßgeblichen Einfluss auf die Lebensqualität der Betroffenen und ihrer Familien. Ein spezielles Ferienlager sollte jungen Nahrungsmittelallergikern in Großbritannien nun dabei helfen, Selbstbewusstsein und Eigenverantwortung aufzubauen. $\mathrm{Ob}$ dies gelang, wurde anschließend anhand von Befragungen evaluiert.
\end{abstract}

D as therapeutische Ziel bei einer Nahrungsmittelallergie, Symptome zu vermeiden, ist nur durch die intensive Auseinandersetzung mit dem Thema zu erreichen: So ist beim Kauf und Verzehr von Nahrungsmitteln permanent Wachsamkeit geboten und - bei schweren Symptomen - eine stetige Notfallbereitschaft und das konsequente Mitführen eines Notfallsets Pflicht. Ohne fremde Hilfe durch Therapeuten, Selbsthilfegruppen und ein unterstützendes Umfeld ist das Krankheitsmanagement nicht oder nur mit Einschränkungen zu leisten. Besonders bei jüngeren Kindern ist es schwierig, aber unbedingt notwendig, Selbstbewusstsein und Eigenverantwortung aufzubauen. In Großbritannien hat die dort ansässige Anaphylaxis Campaign den Versuch gestartet, sich dieser Herausforderung zu stellen, und zwar mit dem Angebot eines einwöchigen Ferienlagers für Betroffene. Ziel dabei war es, im Rahmen eines Aktivurlaubs eine sichere und unterstützende Umgebung außerhalb des häuslichen Wohnumfelds für Kinder mit schweren Nahrungsmittelallergien zu schaffen und den Teilnehmern damit die Gelegenheit zu geben, die Risiken ihrer Nahrungsmittelallergie einzuschätzen und zu bewältigen.

In der vorgestellten Studie wurde untersucht, ob sich die Teilnahme an einem solchen Ferienlager auf verschiedene psychologische und soziale Faktoren auswirkt und ob sie die Kinder befähigt, angemessener mit ihrer Erkrankung umzugehen. Vor und nach Ende des Aktivurlaubs sowie drei und sechs Monate später füllten die Teilnehmer kinderspezifische Fragebögen zur gesundheitsbezogenen Lebensqualität, zur nahrungsmittelallergiespezifischen Lebensqualität, zur Angst und zur Kontrollüberzeugung aus.

24 Kinder (17 Jungen und sieben Mädchen, Elf- und Zwölfährige) mit schwe- ren Nahrungsmittelallergien nahmen an dem Ferienlager teil. Bei allen Kindern lag die Diagnose mindestens ein Jahr zurück, allen war ein Adrenalininjektor verschrieben worden.

Die Auswertung der Fragebögen ergab insgesamt deutliche Verbesserungen hinsichtlich der sozialen und der nahrungsmittelallergiespezifischen Lebensqualität, wobei die gesundheitsbezogene Lebensqualität insgesamt hoch war und sich kaum von den Normdaten unterschied. Die Kontrollüberzeugung insgesamt und die internale Kontrollüberzeugung stiegen bis zum zweiten Follow-up kontinuierlich an. Die Zeit im Ferienlager hatte die Kinder also dazu motiviert, im Laufe der beobachteten Zeit angemessene gesundheitsbezogene Verhaltensweisen anzuerkennen und damit selbst Verantwortung zu übernehmen. Die externale Kontrollüberzeugung, d.h. die Wahrnehmung, dass die Kontrolle ihrer Erkrankung nicht bei Ihnen, sondern bei mächtigen Anderen (z. B. Eltern, Arzt etc.) liegt, nahm dagegen deutlich ab. Auch Angst insgesamt und obsessive Zwangsstörungen verringerten sich im Verlauf, wobei ein Zusammenhang zwischen obsessiven Zwangsstörungen und Nahrungs- mittelallergie bisher nicht beschrieben ist. Eine stärkere Angst war signifikant mit einer geringeren Lebensqualität assoziiert. Bemerkenswert ist, dass der Ausgangswert für Angst insgesamt dem europäischer Kinder (ohne chronische Erkrankung) entsprach, für soziale Angst lag er sogar niedriger, für Panikattacken allerdings deutlich höher. Vor allem in den Teilaspekten Panik, Trennungsangst, soziale Angst und obsessive Zwangsstörungen verringerten sich die Werte über die Zeit des Aktivurlaubs hinaus auf ein Maß deutlich unterhalb der Normgrößen - ein Hinweis auf einen länger anhaltenden Erfolg solcher Maßnahmen.

Knibb RC, Hourihane JO. The psychosocial impact of an activity holiday for young children with severe food allergy: a longitudinal study. Pediatr Allergy Immunol 2013; 24: 368-75

Kommentar: Programme wie diese machen Mut und lassen hoffen, dass sich die starken Einschränkungen hinsichtlich der Lebensqualität - gerade für junge Nahrungsmittelallergiker - maßgeblich verringern lassen. Sie verdeutlichen, wie wichtig es ist, die Betroffenen frühzeitig in das Krankheitsmanagement einzubeziehen, ihnen altersgerecht Verantwortung zu übertragen und ihr Selbstvertrauen zu fördern, statt sie aus Fürsorge in vielen Situationen zu entmündigen. Allerdings brauchen solche Initiativen viel Zeit und Engagement, aber auch eine gute personelle Ausstattung und viel interdisziplinäres fachliches Know-how, um tatsächlich eine unterstützende und sichere Umgebung für die jungen Patienten zu schaffen.

Dr. Imke Reese

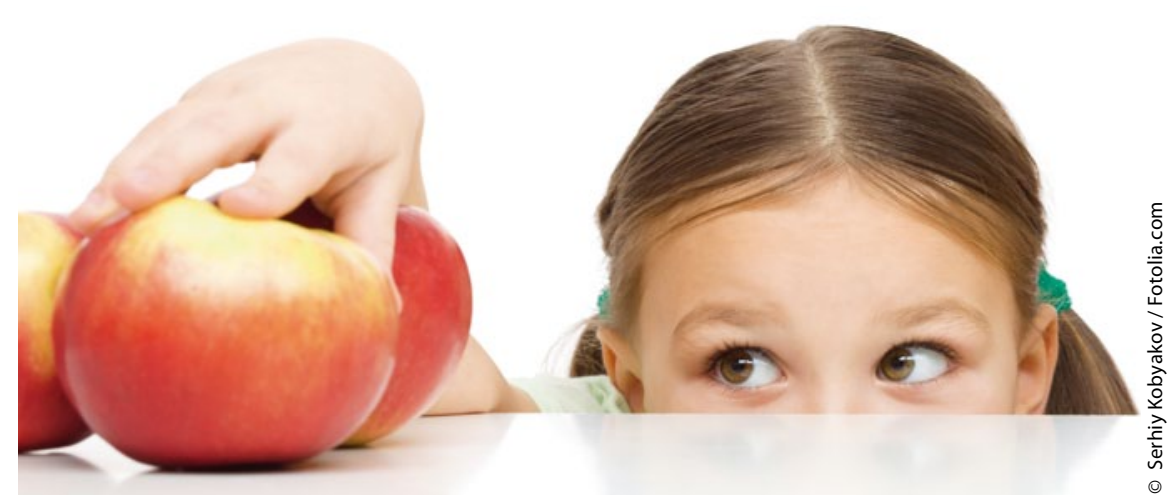

Darf ich oder darf ich nicht? In einem Ferienlager der britischen Anaphylaxis Campaign sollten junge Nahrunsmittelallergiker den Umgang mit ihrer Krankheit lernen. 